\title{
Does Pseudomonas aeruginosa Colonization Affect Exercise Capacity in CF?
}

\author{
Asterios Kampouras, ${ }^{1}$ Elpis Hatziagorou ${ }^{10},{ }^{1}$ Vasiliki Avramidou, ${ }^{1}$ \\ Vasiliki Georgopoulou, ${ }^{2}$ Fotios Kirvassilis, ${ }^{1}$ and John Tsanakas ${ }^{1}$ \\ ${ }^{1}$ Pediatric Pulmonology and CF Unit, $3^{\text {rd }}$ Department of Pediatrics, Hippokration Hospital, Aristotle University of Thessaloniki, \\ Thessaloniki, Greece \\ ${ }^{2}$ Radiology Department, Hippokration Hospital, Thessaloniki, Greece
}

Correspondence should be addressed to Elpis Hatziagorou; elpcon@otenet.gr

Received 23 April 2019; Accepted 9 October 2019; Published 17 December 2019

Academic Editor: Roberto Walter Dal Negro

Copyright ( 2019 Asterios Kampouras et al. This is an open access article distributed under the Creative Commons Attribution License, which permits unrestricted use, distribution, and reproduction in any medium, provided the original work is properly cited.

\begin{abstract}
Introduction. Cardio-Pulmonary Exercise Testing (CPET) has been recognized as a valuable method in assessing disease burden and exercise capacity among CF patients. Aim. To evaluate whether Pseudomonas aeruginosa colonization status affects Exercise Capacity, LCI and High-Resolution Computed Tomography (HRCT) indices among patients with CF; to check if Pseudomonas colonization can predict exercise intolerance. Subjects. Seventy-eight (78) children and adults with CF (31 males) mean (range) age $17.08(6.75 ; 24.25)$ performed spirometry, Multiple Breath Washout (MBW) and CPET along with HRCT on the same day during their admission or follow up visit. Results. 78 CF patients (mean FEV1: 83.3\% mean LCI: 10.9 and mean $\mathrm{VO}_{2}$ peak: $79.1 \%$ ) were evaluated: 33 were chronically colonized with Pseudomonas aeruginosa, 24 were intermittently colonized whereas 21 were Pseudomonas free. Statistically significant differences were observed among the three groups in: peak oxygen uptake \% predicted $\left(\mathrm{VO}_{2}\right.$ peak\% $(p<0.001)$, LCI $(p<0.001)$, as well as FEV1\% $(p<0.001)$ and FVC\% $(p<0.001)$. Pseudomonas colonization could predict $\mathrm{VO}_{2}$ peak\% $\left(p<0.001, r^{2}\right.$ : -0.395$)$. Conclusion. Exercise capacity as reflected by peak oxygen uptake is reduced in Pseudomonas colonized patients and reflects lung structural damages as shown on HRCT. Pseudomonas colonization could predict exercise limitation among CF patients.
\end{abstract}

\section{Introduction}

Cystic Fibrosis lung disease is characterized by the presence and persistence of thick mucus secretions that are not easily cleared from the patient's airways $[1,2]$. This decreased mucus clearance predisposes to early colonization from pathogens, especially Pseudomonas aeruginosa (P. aeruginosa), thus leading to a vicious cycle of infection and inflammation [3]. Pseudomonas colonization has been shown to be a factor influencing FEV1 decline in cystic fibrosis, increasing the hazard of developing severe lung disease to 2.4 times [4] and consequently having a strong impact on overall prognosis.

Since many years, prognosis in CF is best evaluated with Cardiopulmonary Exercise Testing (CPET) $[5,6]$. Exercise testing measures the peak oxygen volume absorbed $\left(\mathrm{VO}_{2}\right.$ peak)by a person's lungs during maximal exercise highlighting his exercise capacity. In $\mathrm{CF}$, patients with $\mathrm{VO}_{2}$ peak values $>80 \%$ predicted have been shown to present nearly excellent 10-year prognosis [5]. Pseudomonas colonization as a factor of inflammation and infection has been shown to affect exercise capacity. Recently, Van de Weert-van Leeuwen et al., have showed that Pseudomonas is an independent factor associated with longitudinal changes in $\mathrm{VO}_{2}$ peak and exercise capacity.

Even though long-term effects of $P$. aeruginosa colonization in $\mathrm{VO}_{2}$ peak have been made profound, no data exist on how exercise capacity of CF patients colonized compares to that of Pseudomonas-free and if such impairment exists, whether it is translated to structural lung damages as seen on High Resolution Computed Tomography (HRCT).

Aim of the present study was to perform a cross-sectional analysis of exercise capacity in CF patients chronically 
TABLE 1: Demographical characteristics of patients participating in the study.

\begin{tabular}{lc}
\hline & Median $(\mathrm{sd}) / N(\%)$ \\
\hline Patients & $78(31$ males $)$ \\
Age & $14.90(4.68)$ \\
Weight $(\mathrm{kg})$ & $47.17(13.44)$ \\
Height $(\mathrm{cm})$ & $153.66(14.34)$ \\
BMI $\left(\mathrm{kg} / \mathrm{m}^{2}\right)$ & $19.57(3.32)$ \\
$\Delta$ F508 homozygous/heterozygous & $20(25.6 \%) / 38(48.7 \%)$ \\
Pancreatic sufficient $(\%)$ & $73(93.6 \%)$ \\
PsA colonized & \\
Intermittently & $24(30.8 \%)$ \\
Chronically & $33(42.3 \%)$ \\
\hline
\end{tabular}

colonized with $P$. aeruginosa, intermittently colonized or free of any pathogen, and also to investigate possible differences in HRCT and whether any correlations with exercise capacity exist.

\section{Materials and Methods}

A total of 78 CF patients mean age 14.9 years old (4.69) attending the CF center of our Department, participated our study. Of these, 73 (93.6\%) were on pancreatic replacement therapy. Intermittently colonized with $P$. aeruginosa were 24 (30.8\%), $33(42.3 \%)$ of them were chronically colonized while 21 were $P$. aeruginosa free.

2.1. Anthropometry. Height and weight were measured in light clothing. Anthropometric data are shown in Table 1.

2.2. Spirometry. Standard spirometry with an electronic spirometer (Vitalograph 2120, Vitalograph Ltd., Ennis, Ireland) according to the ATS/ERS standards [7] was applied in order to estimate Forced Vital Capacity (FVC) and forced expiratory volume in one second (FEV1). Data were expressed in \%predicted using the normative data from the Global Lung Function Initiative software (GLI 2012, Global Lung Function Initiative Task Force, available at: http://www.lungfunction.org/).

2.3. Multiple Breath Washout. MBW measurements were performed with a flow, volume and molecular measurement analyzer (EXHALYZER D, Ecomedics, Switzerland), according to ERS/ATS Consensus Guidelines [8]. LCI is defined as the number of FRC lung turnovers (TO: Cumulated Expired Volume divided by the Functional Residual Capacity (FRC)) required to reduce end-tidal $\mathrm{N}_{2}$ concentration to $1 / 40$ of the starting concentration, which accounts to $2.5 \%$ of the initial one. A pulmonary disease resulting in uneven ventilation distribution, prolongs the duration of the washout, thus elevate the LCI. Normal LCI values are found to be lower than 7 [9, 10]. Moment ratio M1/M0 and M2/M0 emphasize on delayed gas washout due to peripheral airway regions pathology [11]. Ventilation distribution is performed through convection and diffusion. Two other indices have been proposed to express pathology throughout the trancheobrocheal tree: (a) Scond,
TABLE 2: Spirometric, MBW, CPET and Bhalla score values in the 3 patient groups (values are shown as mean (StDev)).

\begin{tabular}{lccc}
\hline & $\begin{array}{c}\text { Free of } P \text {. } \\
\text { aeruginosa }\end{array}$ & $\begin{array}{c}\text { Intermittently } \\
\text { colonized }\end{array}$ & $\begin{array}{c}\text { Chronically } \\
\text { colonized }\end{array}$ \\
\hline FEV1\% pred & $108.0(13.9)$ & $91.6(18.4)$ & $73.1(21.0)$ \\
FVC\% pred & $107.0(17.5)$ & $97.0(17.9)$ & $80.3(18.7)$ \\
$\mathrm{FEF}_{50} \%$ pred & $100.5(16.9)$ & $81.2(30.8)$ & $61.8(30.9)$ \\
$\mathrm{LCI}$ & $8.6(2.2)$ & $10.4(2.5)$ & $13.3(4.0)$ \\
Bhalla score & $4(5.65)$ & $5.5(2.38)$ & $11.00(4.2)$ \\
$\mathrm{VO}_{2}$ peak\% & $91.3(26.3)$ & $83.5(24.0)$ & $68.5(18.3)$ \\
\hline
\end{tabular}

which represents convection-dependent inhomogeneity and (b) Sacin that represents diffusion convection-interactiondependent inhomogeneity [8]. Finally, FRC expresses the remaining air in the lungs at the end of expiration which is in direct relevance with the airway opening $[12,13]$.

2.4. Cardiopulmonary Exercise Testing (CPET). All participants performed a maximal cardiopulmonary exercise testing on a cycle ergometer (Ergoline, Vmax Series V.20-1, Sensor medics). Cardiac parameters were also measured (cardiograph model Corina, S.N. 101164361, Cardiosoft software V5.15). A Gofrey protocol for exercise testing was applied [14]; according to that, depending on subject's height, after baseline measurements for 1 minute and a warm-up period of 2 minutes cycling with 10 watts (for patients $<120 \mathrm{~cm}$ tall), 15 watts $(120-150 \mathrm{~cm})$ or 20 watts $(>150 \mathrm{~cm}$ tall),work load was increased by $10,15,20$ watts respectively every minute until volitional fatigue. Exercise time was kept within 8-12 min. Patient's heart rate over $85 \%$ of maximum predicted [15] along with Respiratory equivalence ratio (RER) over 1.05 [16] were used as indicators of a maximal test. The following parameters were measured: peak oxygen uptake $\left(\mathrm{VO}_{2}\right.$ peak), peak oxygen uptake/weight $\left(\mathrm{VO}_{2}\right.$ peak $\left./ \mathrm{kg}\right)$ ventilator equivalent ratios for oxygen and carbon dioxide at peak exercise $\left(\mathrm{VE} / \mathrm{VO}_{2}, \mathrm{VE} /\right.$ $\mathrm{VCO}_{2}$ ), anaerobic threshold (AT), breathing reserve at peak exercise (BR\%), dead space to exhaled volume ratio $(\mathrm{Vd} / \mathrm{Vt})$. $\mathrm{VO}_{2}$ peak\% predicted was calculated using the Orenstein gender specific equations [17]:

Girls : $\mathrm{VO}_{2} \max (1 / \mathrm{min})=0,0308806 \times$ Height $(\mathrm{cm})-2,877$,

Boys : $\mathrm{VO}_{2} \max (1 / \mathrm{min})=0,044955 \times \operatorname{Height}(\mathrm{cm})-4,64$.

Then the $\mathrm{VO}_{2}$ peak\% predicted was calculated.

Breathing reserve was calculated as: $\mathrm{MVV}-\mathrm{VE} / \mathrm{MVV}$ $(\mathrm{MVV}=$ maximal voluntary ventilation; $(\mathrm{MVV}=35 \times \mathrm{FEV} 1)$; $\mathrm{VE}=$ maximum exercise ventilation). Anaerobic threshold (AT) was determined by the Sensor Medics software, using the $\mathrm{VCO}_{2} / \mathrm{VO}_{2}$ plot.

2.5. HRCT. HRCT scans were performed on an (Asteion) Toshiba CT scanner. Slices measuring $1.5 \mathrm{~mm}$ were obtained at $10 \mathrm{~mm}$ intervals during suspended respiration in supine position. Additional expiratory scans were obtained in older cooperative 


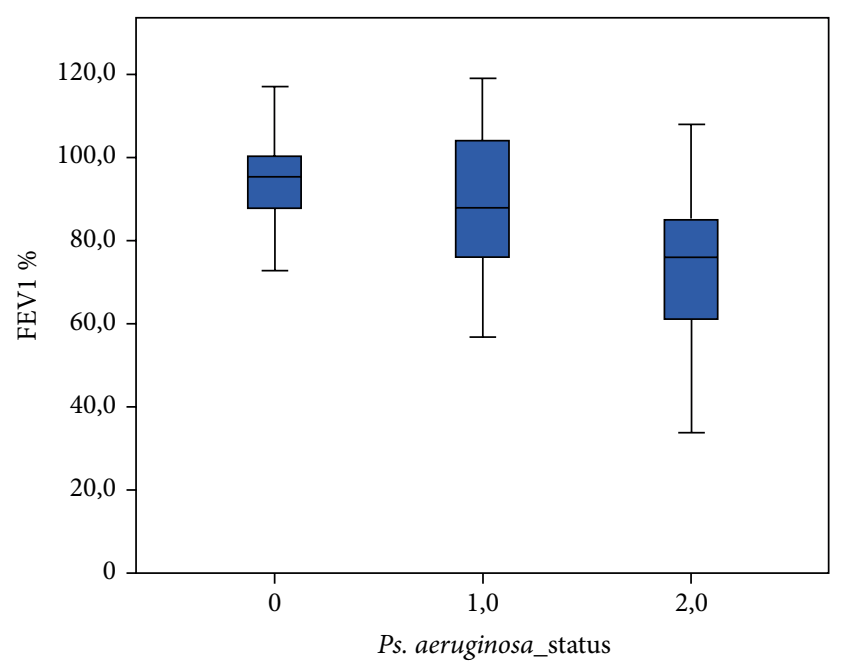

FIGURE 1: FEV1\% predicted among the three groups of patients. (0: patients not colonized, 1: patients intermittently colonized with $P$. aeruginosa, 2: patients chronically colonized with $P$. aeruginosa).

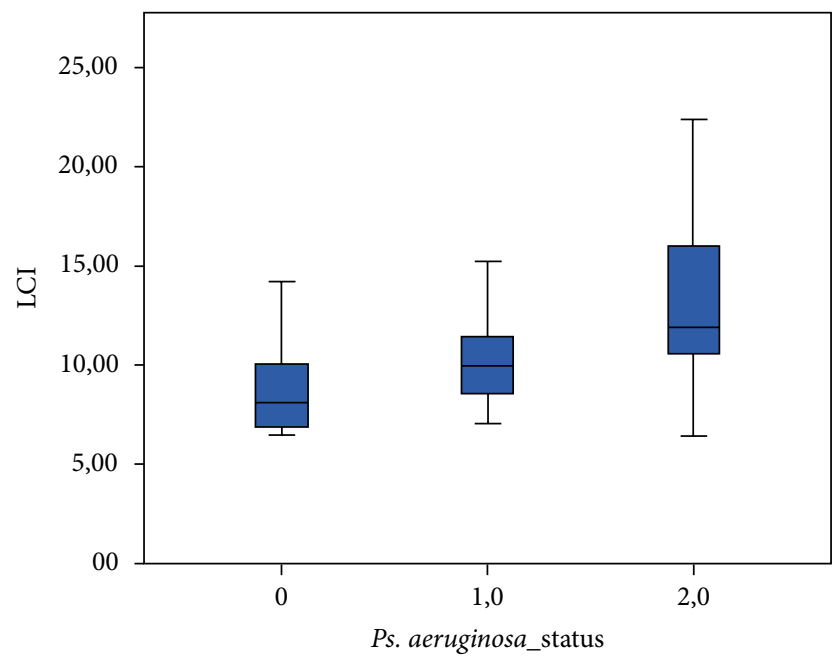

FigURE 2: LCI among the three groups of patients. (0: patients not colonized, 1: patients intermittently colonized with $P$. aeruginosa, 2: patients chronically colonized with $P$. aeruginosa).

children at $20 \mathrm{~mm}$ intervals. Each CT scan was scored using the "Bhalla" scoring system by an experienced radiologist; the Bhalla score shows high inter-observer reproducibility and sensitivity $[18,19]$. The following changes were evaluated: severity and extent of bronchiectasis, severity of peri-bronchial thickening, generation of bronchial division involved, extent of mucus plugging, sacculation or abscess formation, bullae, emphysema, atelectasis and consolidation. Higher values of the Bhalla score indicate more severe lung disease.

2.6. Statistical Analysis. Descriptive statistics were used to describe study population. All parameters were described as mean and standard deviation (sd). Kolmogorov-Smirnov test was applied to express normality while Spearman's Correlation Coefficients were used to assess for possible correlations

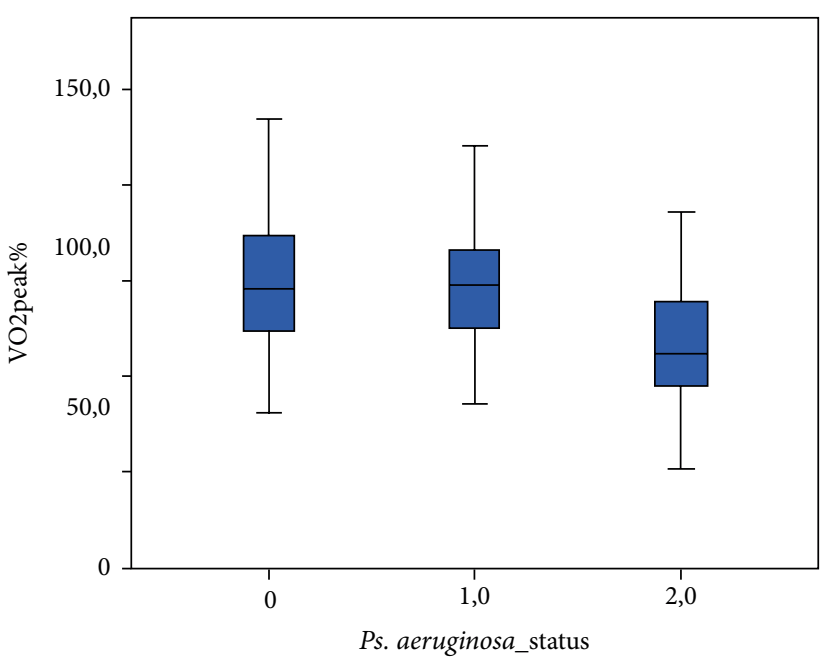

FIGURE 3: $\mathrm{VO}_{2}$ peak\% predicted among patients not colonized, intermittently and chronically colonized with Pseudomonas aeruginosa. (0: patients not colonized, 1: patients intermittently colonized with $P$. aeruginosa, 2: patients chronically colonized with P. aeruginosa).

between above parameters. Correlation coefficient values $<0.10$ represent weak correlation, 0.20 weak to moderate, $0.25-0.35$ moderate correlation, 0.40 moderate to strong whereas values $>0.50$ were indicative of strong correlation [20].

\section{Results}

In total $78 \mathrm{CF}$ patients mean age (sd) $14.9(4.68)$ years participated. Mean FEV1 (\%predicted) was $87.5 \%$, mean LCI 10.87 and mean $\mathrm{VO}_{2}$ peak\% predicted was $79.1 \%$. Of all patients, intermittently colonized with $P$. aeruginosa were $24(30.8 \%)$, $33(42.3 \%)$ of them were chronically colonized while 21 were $P$. aeruginosa free.

Spirometric, MBW, CPET and Bhalla score values among the three patient groups are shown in Table 2. FEV1 (\%predicted) differed significantly ( $p \leq 0.001)$ between the three groups (Figure 1). There was a significant difference noted for the LCI (Figure 2), while patients chronically, intermittently and not colonized with Pseudomonas aeruginosa presented significant differences concerning their $\mathrm{VO}_{2}$ peak. $(p<0.001)$ (Figures 3 and 4).

When regression analysis was applied, Pseudomonas status was shown to be a strong predictor of exercise limitation in CF patients $\left(p<0.0001, r^{2}:-0.395\right)$.

\section{Discussion}

The main finding of this study is that $P$. aeruginosa colonization is always indicative of exercise intolerance and thus impaired activity in CF even if the patient's respiratory function is preserved.

During the past years progress in the treatment of Cystic Fibrosis has led to an overall increase in the expected life span for CF patients [21]. This improved prognosis has shifted 


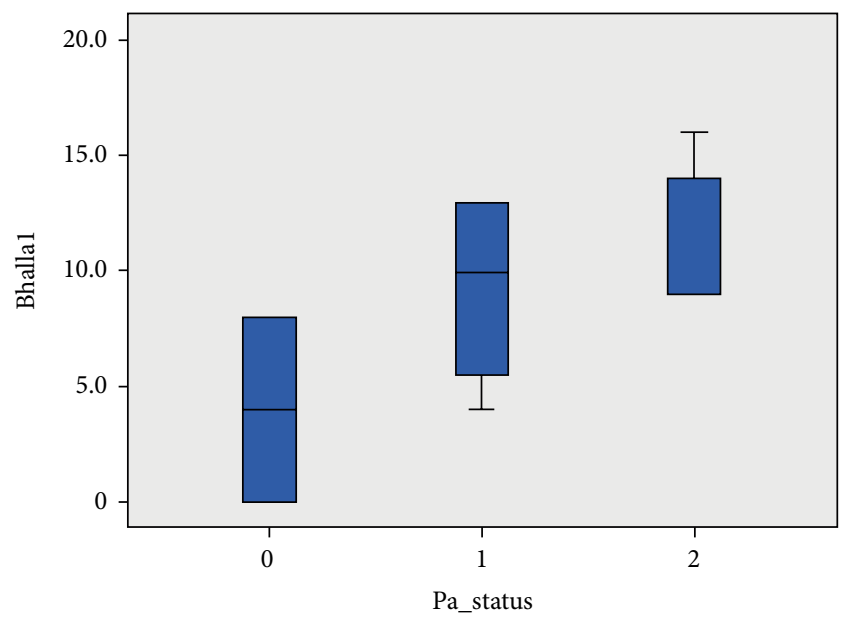

FIGURE 4: Bhalla scores among patients not colonized, intermittently and chronically colonized with Pseudomonas aeruginosa. (0: patients not colonized, 1: patients intermittently colonized with $P$. aeruginosa, 2: patients chronically colonized with $P$. aeruginosa.

attention to improving quality of life especially in the adolescent and adult CF population. Major determinant of quality of life is exercise capacity-the maximum physical exertion that a person can sustain.

Exercise capacity is at best evaluated with a full Cardiopulmonary Exercise Test (CPET). Peak oxygen uptake upon maximal exercise $\left(\mathrm{VO}_{2}\right.$ peak) has been shown to be a significant predictor of life expectancy not only in CF patients but also in healthy population [22]. Even though CPET can provide valuable information regarding a patient's status, few CF centers worldwide implement exercise testing as a part of their routine CF evaluation $[23,24]$.

In the present study, we showed that patients colonized with $P$. aeruginosa have impaired exercise capacity and Lung Clearance index in contrast to noncolonized. Apart from that, it was also shown that chronic colonization affects exercise capacity even more than intermittent colonization and can predict structural lung damages, along with functional capacity of CF patients.

Chronic $P$. aeruginosa infection leads to a vicious cycle of inflammation and subsequent airway remodeling $[25,26]$. These structural lung damages in CF patients lead to impaired gas exchange [27]. Even though this pathway to impaired gas exchange has been nearly clarified, no studies were performed before trying to look into the causality effect of Pseudomonas status on HRCT abnormalities. In our study, $P$. aeruginosa colonization was proven to be a prognostic factor of structural lung damages and an index of impaired exercise capacity. Thus, our study fills a gap in existing literature highlighting the fact that Pseudomonas can be an indicator of impaired exercise capacity which can be followed by alterations in HRCT.

Our finding comes in accordance to the findings of Van de Weert-van Leeuwen et al., who showed that longitudinally, Pseudomonas colonization can affect exercise capacity. The fact that Pseudomonas colonization is indicative of impaired exercise capacity and thus an additional burden in everyday activities of CF patients can be helpful to CF centers that are not able to perform exercise testing. Physicians with patients colonized intermittently or chronically could use this knowledge to prescribe exercise rehabilitation even in an early stage in order for daily activities to not be hassled [17].

A few limitations should be taken into consideration though. Even though our study looks into $\mathrm{VO}_{2}$ peak and Bhalla score in patients with preserved respiratory function colonized or not, differences in FEV1\% predicted and LCI between groups were significant. Having three groups with similar respiratory function would lead to safer results. However, as Pseudomonas status affects respiratory function in CF this could not be possible in our study nor the adjustment for it. Perhaps a study with larger cohorts could clarify this. Additionally, even though the effects of $P$. aeruginos a colonization on exercise capacity seem obvious, no studies before have looked into the extent of this effect nor correlate it with structural damages as shown on HRCT.

In conclusion, Pseudomonas aeruginosa colonization in CF youths is well associated with structural lung damages and impaired exercise capacity. Actions to improve activity status should be taken as soon as colonization is known.

\section{Data Availability}

The data used to support the findings of this study were supplied by Elpis Hatziagorou. Requests for access to these data should be made to [Elpis Hatziagorou, ehatziagorou@gmail. com].

\section{Ethical Approval}

Aristotle University of Thessaloniki Medical School Ethics Committee (3/2-5-2018).

\section{Conflicts of Interest}

All authors would like to confirm that there is nothing to declare.

\section{Acknowledgments}

The manuscript has been presented in the European Respiratory Society Conference 2017, https://erj.ersjournals. com/content/50/suppl_61/PA1837.

\section{References}

[1] J. C. Davies, E. W. Alton, and A. Bush, "Cystic fibrosis," BMJ, vol. 335, no. 7632, pp. 1255-1259, 2007.2007-12-13 23:01:06

[2] J. F. TomashefskiJr. and C. R. Abramowsky, "The pathology of cystic fibrosis," Cystic Fibrosis, Marcel Dekker Inc, New YorkP. B. Davis, Ed., , 1993.

[3] R. L. B. J. Gibson and B. W. Ramsey, "Pathophysiology and management of pulmonary infections in cystic fibrosis," American Journal of Respiratory and Critical Care Medicine, vol. 168, no. 8, pp. 918-951, 2003. 
[4] E. Kerem, L. Viviani, A. Zolin et al., "Factors associated with FEV1 decline in cystic fibrosis: analysis of the ECFS patient registry," European Respiratory Journal, vol. 43, no. 1, pp. 125133, 2014.

[5] P. A. Nixon, D. M. Orenstein, S. F. Kelsey, and C. F. Doershuk, "The prognostic value of exercise testing in patients with cystic fibrosis," New England Journal of Medicine, vol. 327, no. 25, pp. 1785-1788, 1992.

[6] S. L. S. Nguyen, C. Cracowski, T. Perez et al., "Prognostic value of clinical exercise testing in adult patients with cystic fibrosis," Revue des Maladies Respiratoires, vol. 27, no. 3, pp. 219-225, 2010.

[7] N. Beydon, S. D. Davis, E. Lombardi et al., "An official American Thoracic Society/European Respiratory Society statement: pulmonary function testing in preschool children," American Journal of Respiratory and Critical Care Medicine, vol. 175, no. 12, pp. 1304-1345, 2007.

[8] P. D. Robinson, P. Latzin, S. Verbanck et al., "Consensus statement for inert gas washout measurement using multipleand single- breath tests," European Respiratory Journal, vol. 41, no. 3, pp. 507-522, 2013.

[9] S. I. Fuchs and M. Gappa, "Lung clearance index: clinical and research applications in children," Paediatric Respiratory Reviews, vol. 12, no. 4, pp. 264-270, 2011.

[10] S. Yammine, F. Singer, C. Abbas, M. Roos, and P. Latzin, "Multiple-breath washout measurements can be significantly shortened in children," Thorax, vol. 68, no. 6, pp. 586-587, 2013.

[11] S. Stanojevic, R. Jensen, D. Sundaralingam et al., "Alternative outcomes for the multiple breath washout in children with CF," Journal of Cystic Fibrosis, vol. 14, no. 4, pp. 490-496, 2015.

[12] P. M. Gustafsson, H. J. Johansson, and G. O. Dahlbäck, "Pneumotachographic nitrogen washout method for measurement of the volume of trapped gas in the lungs," Pediatric Pulmonology, vol. 17, no. 4, pp. 258-268, 1994.

[13] P. M. Gustafsson, S. Källman, H. Ljungberg, and A. Lindblad, "Method for assessment of volume of trapped gas in infants during multiple-breath inert gas washout," Pediatric Pulmonology, vol. 35, no. 1, pp. 42-49, 2003.

[14] S. Godfrey, C. T. Davies, E. Wozniak, and C. A. Barnes, "Cardiorespiratory response to exercise in normal children," Clinical Science, vol. 40, no. 5, pp. 419-431, 1971.

[15] ATS/ACCP, "Statement on cardiopulmonary exercise testing," American Journal of Respiratory and Critical Care Medicine, vol. 167, no. 2, pp. 211-277, 2003.

[16] D. Orenstein, "Assessment of exercise pulmonary function," Pediatric Laboratory Exercise Testing Clinical Guidelines, Human Kinetics, ChampaignT. W. Rowland, Ed., pp. 141-163, 1993.

[17] V. Avramidou, E. Hatziagorou, A. Kampouras et al., "Lung clearance index (LCI) as a predictor of exercise limitation among CF patients," Pediatric Pulmonology, vol. 53, no. 1, pp. 81-87, 2018.

[18] S. I. Fuchs, M. Gappa, J. Eder, K. M. Unsinn, G. Steinkamp, and H. Ellemunter, "Tracking lung clearance index and chest CT in mild cystic fibrosis lung disease over a period of three years," Respiratory Medicine, vol. 108, no. 6, pp. 865-874, 2014.

[19] F. Cademartiri, G. Luccichenti, A. A. Palumbo et al., "Predictive value of chest CT in patients with cystic fibrosis: a single-center 10-year experience," AJR American Journal of Roentgenology, vol. 190, no. 6, pp. 1475-1480, 2008.
[20] A. Rubin, Statistics for Evidence-Based Practice and Evaluation, University of Houston, 3rd edition, 2013.

[21] S. Corriveau, J. Sykes, and A. L. Stephenson, "Cystic fibrosis survival: the changing epidemiology," Current Opinion in Pulmonary Medicine, vol. 24, no. 6, pp. 574-578, 2018.

[22] B. Strasser and M. Burtscher, "Survival of the fittest: $\mathrm{VO}_{2}$ max, a key predictor of longevity?," Frontiers in Bioscience (Landmark Edition), vol. 23, pp. 1505-1516, 2018.

[23] M. Barker, A. Hebestreit, W. Gruber, and H. Hebestreit, "Exercise testing and training in German CF centers," Pediatric Pulmonology, vol. 37, no. 4, pp. 351-355, 2004.

[24] D. Stevens, P. J. Oades, N. Armstrong, and C. A. Williams, "A survey of exercise testing and training in UK cystic fibrosis clinics," Journal of Cystic Fibrosis: Official Journal of the European Cystic Fibrosis Society, vol. 9, no. 5, pp. 302-306, 2010.

[25] S. de Bentzmann, P. Roger, and E. Puchelle, "Pseudomonas aeruginosa adherence to remodelling respiratory epithelium," European Respiratory Journal, vol. 9, no. 10, pp. 2145-2150, 1996.

[26] D. Adam, J. Roux-Delrieu, E. Luczka et al., "Cystic fibrosis airway epithelium remodelling: involvement of inflammation," The Journal of Pathology, vol. 235, no. 3, pp. 408-419, 2015.

[27] E. Hatziagorou, A. Kampouras, V. Avramidou et al., "Exercise responses are related to structural lung damage in $\mathrm{CF}$ pulmonary disease," Pediatric Pulmonology, vol. 51, no. 9, pp. 914-920, 2016. 


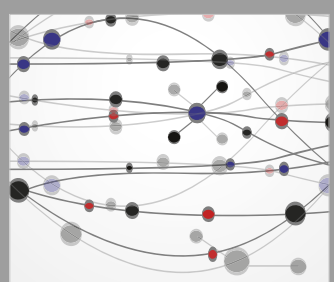

The Scientific World Journal
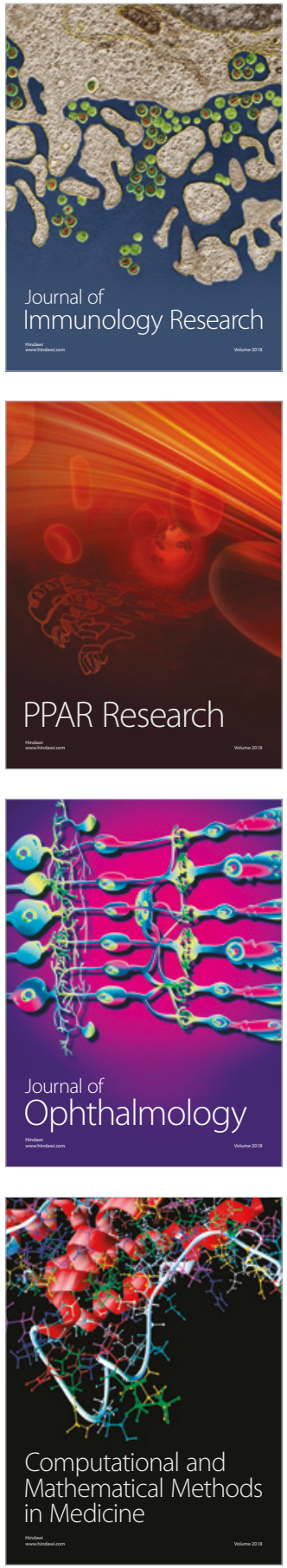

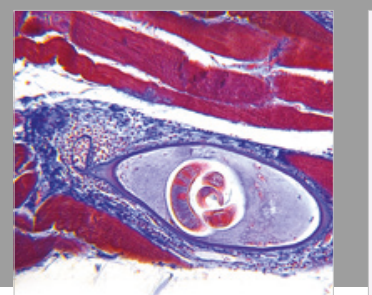

Gastroenterology Research and Practice

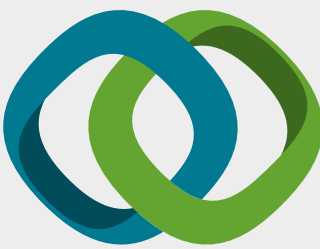

\section{Hindawi}

Submit your manuscripts at

www.hindawi.com
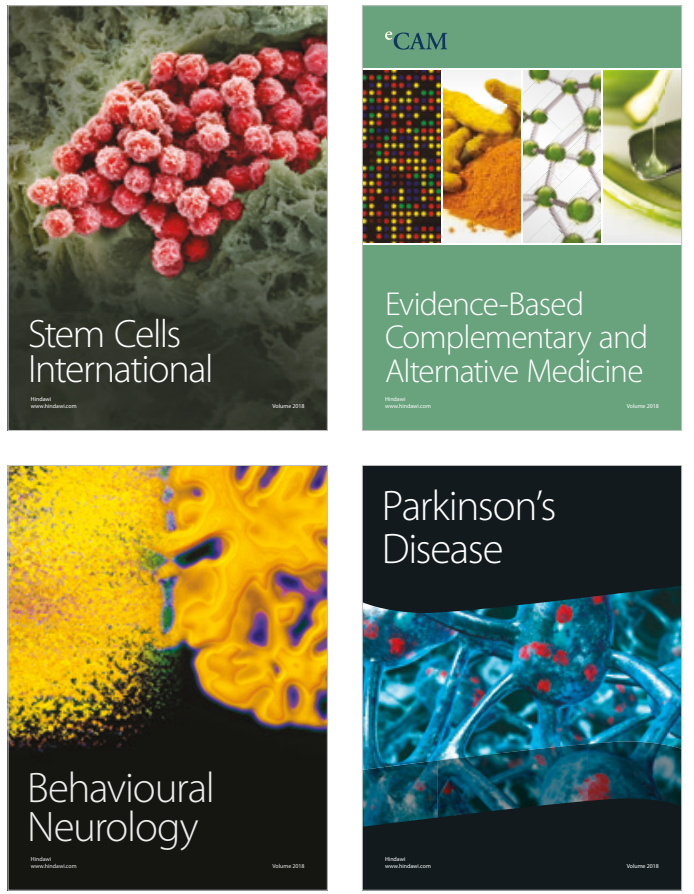

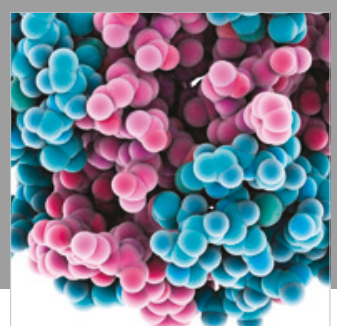

ournal of

Diabetes Research

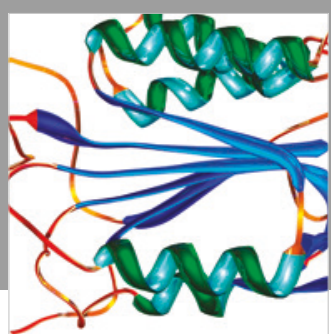

Disease Markers
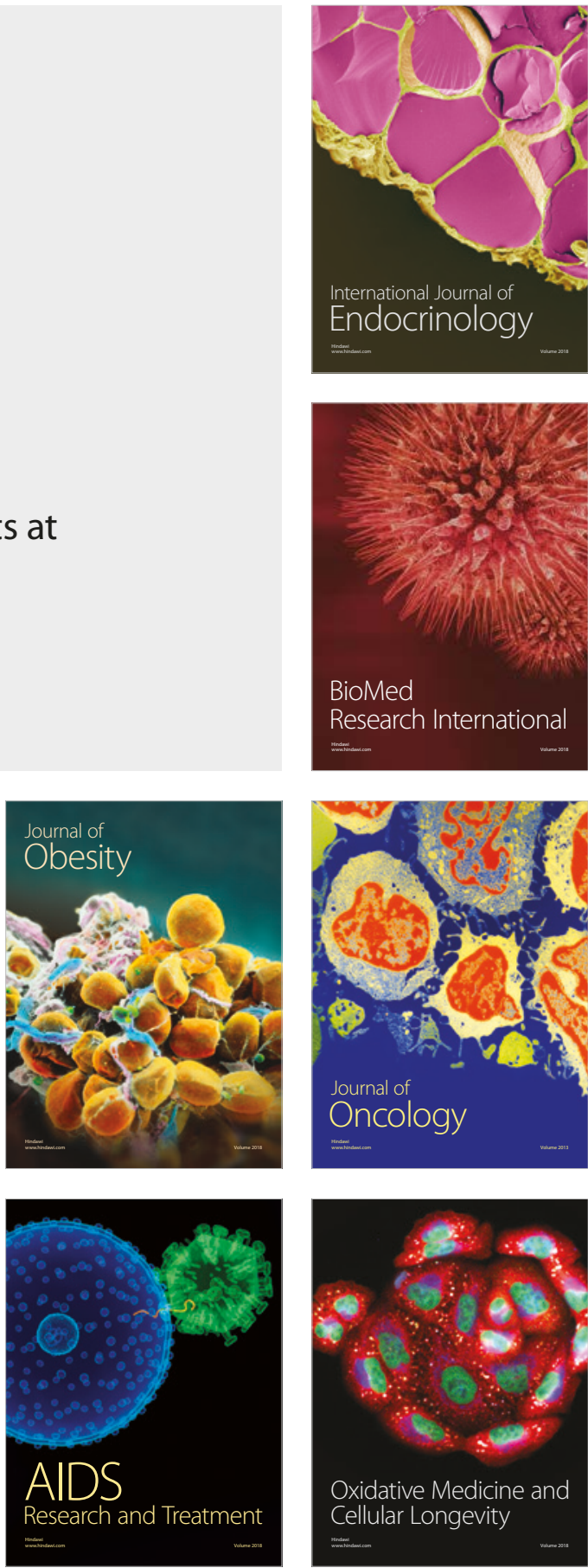